\title{
CAN AHP FACILITATE CONFLICT RESOLUTION? QUANTITATIVE EVIDENCE FROM ROLE PLAYING IN A BUSINESS ENGINEERING CLASS.
}

\author{
Maria Assunta Barchiesi*, Roberta Costa, Marco Greco \\ Department of Business Engineering \\ University of Rome "Tor Vergata" \\ Rome, Italy \\ E-mail addresses: barchiesi@disp.uniroma2.it; costa@disp.uniroma2.it; greco@disp.uniroma2.it
}

\begin{abstract}
The theme of conflict resolution using Analytic Hierarchy Process (AHP) is widely examined in scientific literature, but as yet insufficient attention has been paid to the quantitative aspect of AHP contribution. In this paper we aim to quantify AHP helpfulness in conflict negotiations: how much does AHP actually facilitate a conflict resolution and help negotiators to find $w$ in-w in agreements?

Following this lead, we realized a role playing experiment simulating a "Union versus Management" negotiation problem and compared two groups: a control group that negotiated without any structured decision support system and another group that negotiated with the support of AHP. In order to compare the agreements obtained by means of negotiation and AHP, we acted as an arbiter in the AHP decision process, following Saaty's assumptions on the psychological attitudes of negotiating parties in a conflict. The results showed that AHP agreements are more frequently Pareto-efficient than those achieved by negotiators, and are characterized by higher average utilities.
\end{abstract}

Keywords: AHP, Negotiation, Conflict Resolution, Decis ion Making, Experimenta 1 Economics

\section{Introduction}

Negotiation and conflict resolution using Analytic Hierarchy Process have been studied and applied in many research fields, from construction management to politics. Previous studies suggest that AHP might help to quantify the perception of gains and losses for the parties involved and analyze the issues scientifically, in a more logical manner (Saaty, and Alexander, 1989; Hashem \& Varghese, 2004). Nonetheless, existing research on AHP has not focused on quantitative measure of the effects of its application to conflict resolution: facilitating the conflict resolution and helping negotiators to find winwin agreements.

We carried out a role playing experiment involving Business Engineering students. They simulated a "Union versus Management" negotiation problem: a control group negotiated without any structured decision support system, while the rest of students negotiated with the support of AHP. Each participant has been assigned a role as representative of the Union or of the Management. We provided them with an individual qua litative description of the negotiation background, which was different for the two roles, in order to make easier the identification of the partic ipant with his/her assigned role. We introduced in both descriptions conflict details on the subjective perceptions of the opponents such as strike threats in spite of important deadlines and past questionable behavior of the management. Parties had to negotiate on four different potential concessions/demands: productivity bonus (PB), flexible schedules (FS), increase in break times (BT) and increase in training (T). The utility outcome of a bundle of concessions/demands is different from the sum of the concessions alone, and different for the two parties too.

\footnotetext{
* Corresponding author
} 


\section{Win-win agreements: how to determine feasible sets}

In order to compare the agreements obtained by means of negation and AHP, we acted as an arbiter in the AHP decision process. Our approach is based on Saaty's assumptions (1990) on the psychological attitudes of negotiating parties in a conflict:

- what a party gains from its demands should be greater or at last equivalent to the losses incurred in meeting its opponents demands,

- parties are more likely to find an agreement if they perceive that demands are fairly met.

In the methodology we propose, the arbiter utilizes the values of gains and losses of the conflicting parties, calculated by the AHP model described in the next paragraph, in order to determine what sets of demands are acceptable to all the subjects involved.

Saaty (1990) be lieves that in negotiations each party considers as a gain not only its incremental benefits, but also the costs sustained by its opponent. Each side of the negotiation assesses:

- its gain as the product of its benefits and the opponent (perceived) costs,

- its loss as the product of its costs and the opponent (perceived) benefits.

The ratios for two parties A and B are determined as follow (Saaty, 1990):

$$
\begin{aligned}
& A_{\text {raio }}=\frac{\text { Gain to A }}{\text { A's perception of gain to B }}=\frac{\sum \text { A's benefits } \times \text { B's costs }}{\sum \text { B's benefits } \times \text { A's costs }}=\frac{\text { Gain to A }}{\text { Loss to A }} \\
& B_{\text {ratio }}=\frac{\text { Gain to B }}{\text { B's perception of gain to A }}=\frac{\sum \text { B's benefits } \times \text { A's costs }}{\sum \text { A's benefits } \times \text { B's costs }}=\frac{\text { Gain to B }}{\text { Loss to B }}
\end{aligned}
$$

Following this assumption, each party tries to increase its gain by negotiating in order to increase its benefits and the opponent costs. In the same way, each party tries to reduce its loss by negotiating in order to decrease its costs and the opponent benefits.

On this basis, the arbiter considers the benefits and costs for both parties and he/she determines a feasible agreement. The selected combination of concessions/demands shows each party that the outcome is fair if:

- their gain/loss ratios are more than one,

- their net gains are close.

The arbiter analyses the disparity between admissible solutions (ratios greater than one) and he/she selects the most reasonable resolution of the conflict between solutions that are not dominated, minimizing the difference between parties' net gains.

\section{Facilitating conflict resolution: an application of AHP}

AHP aids to break down the negotiation problem into a multilevel hierarchy structure of objectives, criteria, sub criteria and alternatives. According to a well defined procedure, people involved in the experiment are asked to structure:

- their own actual benefits and costs hierarchies;

- their perceived benefits and costs hierarchies of the other party.

The alternatives were the possible concessions/demands: PB, FS, BT and T. For each of them the participants had to list separately in a table the certain and the uncertain advantages and disadvantages. For example as productivity bonus (PB) advantages and disadvantages the representative of the Union could write the certain disadvantage "increasing working load", the uncertain advantage "economic benefit" (the bonus will be paid only if the productivity objective is achieved), and so on, according to his own ideas about the behavior and the attitude of the role he was playing. The same advantage or disadvantage could appear in different entries of the table (i.e. FS and BT could have the same certain 
advantage "better working life"). In order to facilitate the identification process a table listing possible advantages or disadvantages was provided, but no limits or restrictions were applied to the participants. The only recommendation made was to consider enough relevant elements to represent the problem, avoiding insignificant details. Participants were also asked to fill in another table - identical to the previous one - considering their own perception about the certain and uncertain advantages and disadvantages of the other party.

According the detected tables, for each participant the arbitrator structured four hierarchies: the actual benefits, the actual costs, the perceived benefits of the other party, the perceived costs of the other party. Each hierarchy presented four levels as shown in figures 1 and 2:

1. the goal (to determine actual/perceived benefits or costs);

2. the criteria: certain and uncertain advantages for the benefits hierarchies and certain and uncertain disadvantages for the costs hierarchies;

3. the sub criteria: the entire list of certain and uncertain advantages and disadvantages (obviously in the related hierarchy and connected to the related parent node);

4. the alternatives: PB, FS, BT and T.

According to their own four hierarchies, the participants made comparisons between elements of comparable sets using Saaty scale of importance.

After analyzing the level of inconsistency in the judgments the alternatives were ranked and the relative magnitudes were expressed for actual and perceived benefits and costs.

The application of AHP we propose allowed the comparison of the parties' benefits and costs regardless of the individual representation of the negotiation problem.

Goal: to determine actual/perceived benefits
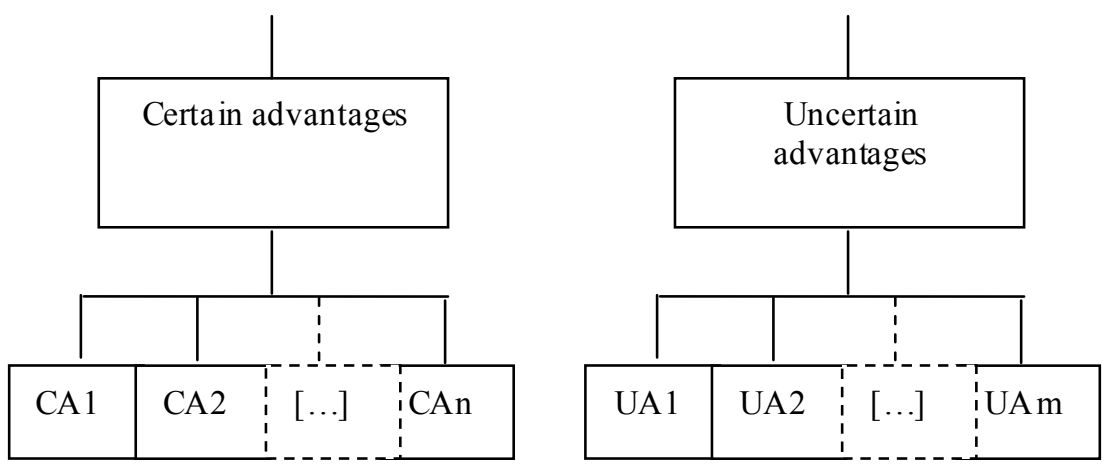

Each concession/demand be low is connected to every subcriterion

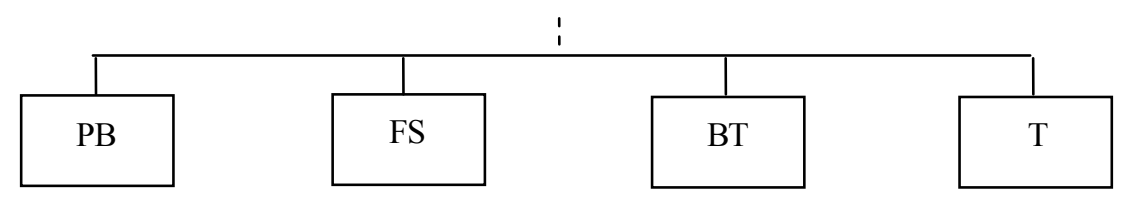

Figure 1: the actual/perceived benefits hierarchy 
Goal: to determine actual/perceived costs
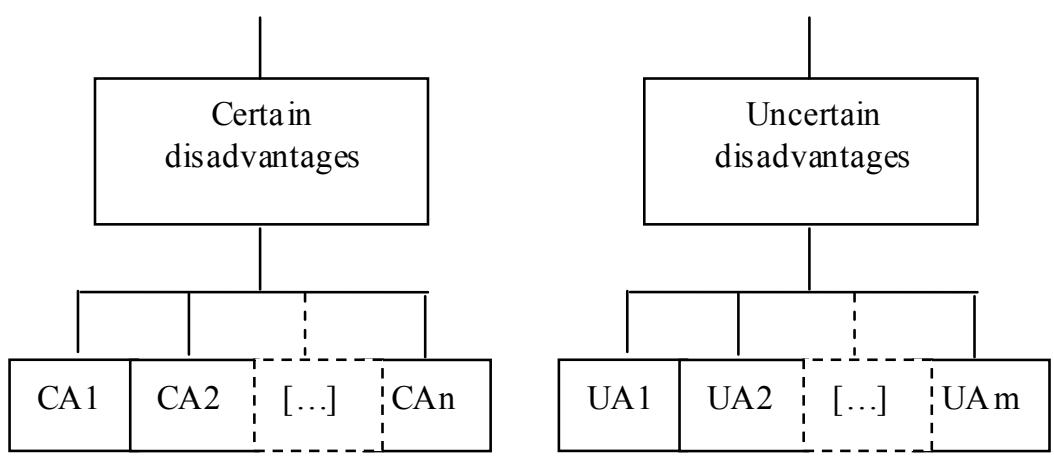

Each concession/demand be low is connected to every subcriteria

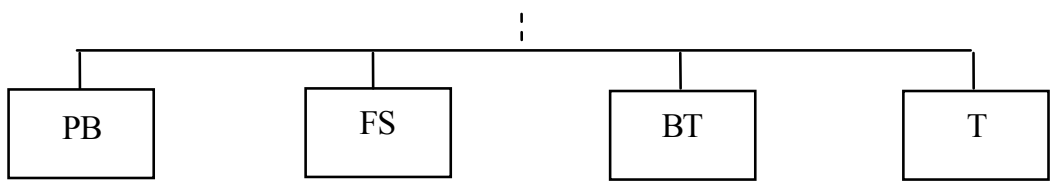

Figure 2: the actual/perceived costs hierarchy

\section{Results}

We compared the results of the negotiation and AHP samples, calculating the utility for each player and identifying Pareto efficient agreements. Utilities are normalized in the range [0, 1]. On average, "negotiators" achieved 0.78 points ( 0.89 for Management, 0.68 for Union), while "AHP players" achieved 0.79 points ( 0.87 for Man., 0.70 for Uni.). A non-parametric Mann-Whitney U-test for the two independent samples of results shows that the Null Hypothesis cannot be rejected. Thus, the distributions of both groups might be equal.

Table 1. Number of Pareto efficient agreements in the two groups.

\begin{tabular}{|l|l|l|}
\hline & Pareto efficient & Not efficient \\
\hline AHP & 5 & 4 \\
\hline Negotiation & 3 & 6 \\
\hline
\end{tabular}

Table 1 shows that AHP helps in finding Pareto efficient agreements with respect to traditional negotiation processes. However, a chi-square test reveals that such difference is not statistically signif icant.

After each negotiation, players have been interviewed about the dynamics of their interactions. In particular, we verified whether the players shared their information or not. As a result, in two couples negotiators behaved cooperatively, sharing their personal utility functions and negotiating under symmetric information conditions. Not surprisingly they both achieved Pareto efficient agreements and high utility scores. 
To make the experiment as realistic as possible the arbiter could not use utility functions in order to improve the imposed agreement, as we hypothesized such functions to be implicit and unknown to him/her.

Moreover, in conflict situation it is not very likely that parties will mutually disclose all the ir private information. If we exclude the two couples of cooperating negotiators, results change slightly. On average, "non-cooperative negotiators" achieved 0.74 points ( 0.86 for Management, 0.63 for Union). According to a Mann-Whitney U-test, the median of the AHP-based distribution is likely to be higher than that of "non-cooperative negotiators" group with $\mathrm{p}=0.11$ (single tailed). Moreover, as shown in Table 2 a chi-square test highlights that the arbiter solutions are Pareto-efficient more frequently than those achieved by non-cooperative negotiators in a statistically significant way $(\mathrm{p}=0.08)$.

Table 2. Number of Pareto efficient agreements in the two groups, excluding "fully cooperative couples".

\begin{tabular}{|l|l|l|}
\hline & Pareto efficient & Not efficient \\
\hline AHP & 5 & 4 \\
\hline Negotiation (non-cooperative) & 1 & 6 \\
\hline
\end{tabular}

\section{Conclusions}

The aim of this paper is to find a quantitative measure which describes the helpfulness of the AHP in conflict negotiations. We sought to answer the following research question: how much does AHP actually facilitate a conflict resolution and help negotiators to find win-win agreements? We simulated a negotiation problem and compared two groups: a control group that negotiated without any structured decision support system and another one that negotiated with the support of AHP.

We found that, on equal terms of non-cooperative situation, agreements imposed by the AHP-based arbiter are more frequently Pareto-efficient than those achieved by negotiators and average utilities achieved by AHP-based solutions are higher than the other group ones in a statistically significant way. Our results might be improved by increasing the cardinality of the sample and the likelihood of the players identifying with their roles, for example by providing more information and more time.

As a future development we will replicate the experiment allowing players to draw their own utility functions on the possible agreement outcomes, according to the provided background information. These further experiments might explore the relation between AHP and the bounded rationality.

\section{ESSENTIAL REFERENCES}

Hashem, M.A., \& Varghese, P.T. (2004). Negotiation and resolution of conflict using AHP: an application to project management. Engineering, Construction and Architectural Management, 11(2), 90100.

Saaty, T.L. (1990). The Analytic Hierarchy Process in Conflict Management. The International Journal of Conflict Management, 1(1), 47-68.

Saaty, T.L., \& Alexander, J. (1989). Conflict Resolution. Praeger, New York, NY. 